\title{
VERTICAL DISTRIBUTION OF NEARSHORE FLOW VELOCITY
}

\author{
Li Chunhui ${ }^{1,2}$ \\ Fu Xiaoyan ${ }^{1,2}$ \\ Li Ruijie ${ }^{1,2}$ \\ Dai Lu ${ }^{1,2}$ \\ Chen Peng ${ }^{1,2}$ \\ ${ }^{1}$ Key Laboratory of Coastal Disaster and Defense, Ministry of Education, Hohai University, \\ Nanjing 210098, China \\ ${ }^{2}$ Environment Marine Laboratory, Hohai University, Nanjing, China
}

\begin{abstract}
In this paper, a new exponential formed vertical distribution of nearshore flow velocity is constructed, which is simpler in form and more suitable for engineering application. The physical meaning of the new formula is more specific than that of Soulsby. Compared with those logarithmic formed ones, the new one does not need the maximum velocity and only needs the mean velocity in vertical, which gives it better engineering practicability. Apply the new formula to Jiangsu coastal area and compare the results with that of Soulsby whose results show the new formula agrees better with the measured flow velocity, which reasonably reflects the basic principles of vertical distribution of flow velocity.
\end{abstract}

Keywords: vertical distribution of flow velocity; Jiangsu Sea; nearshore; exponential formula

\section{INTRODUCTION}

The vertical distribution of nearshore flow velocity is an important issue, which is meaningful to oceanography and ocean engineering. Many scholars have been studying on vertical distribution of flow velocity and a variety of formulae are brought forward, such as Prandtl who put forward a logarithmic one by establishing the theory of mixing length [6], Monin [5], Taylor [13], Schauer [8], Soulsby [11], Mattheus[10] and Song Zhiyao [9] who improved Prandtl formula and Karman and Prandtl[13] who proposed an exponential one by dimensional analysis. And Kumbhakar M[3], Xiao Qianlu[16], Jakubowski[15] and He Bingqu[2] also did several studies on it. Most of these formulae are complex in forms and not convenient for application. In 1990, Soulsby[12] brought forward an empirical formula which has been widely used. However it is not distinct enough in physical meaning and it is a piecewise function, which makes it inconvenient to use. So it is necessary to put forward a new formula for vertical distribution of flow velocity which is simpler, relatively more accurate with definite physical meaning and easier in application.

\section{DERIVATION OF VERTICAL DISTRIBUTION OF FLOW VELOCITY}

$\mathrm{Ni}$ Jinren et al. [6] found a basic formula after summarizing a variety of velocity profile formulae:

$$
F\left(\frac{u}{u_{*}}, \ln \frac{h}{z}\right)=f\left(x_{1}, x_{2} \ldots\right)
$$

Where, $f\left(x_{1}, x_{2} \cdots\right)$ is an undetermined function; $u$ is flow velocity; $u_{*}$ is friction velocity; $h$ is water depth; $z$ is the height above seabed.

A new formula is given through referring to a formula put 
forward by Li Ruijie (2012)[4] and the corrective methods for Prandtl formula:

$$
\ln \frac{u}{u_{*}}=A_{1} \ln \left(\frac{h}{z}\right)+A_{2} \ln z+A_{3}
$$

Where, $A_{1}, A_{2}, A_{3}$ are undetermined parameters, and formula (2) could be written as:

$$
u=u_{*}\left(\frac{h}{z}\right)^{A_{1}} z^{A_{2}} e^{A_{3}}
$$

According to Prandtl mixing length theory, the friction velocity $u_{*}$ could be written as (Fang Guo-hong)[1]:

$$
u_{*}=\frac{\kappa U}{\ln \left(4 h / z_{0}\right)-8 / 3}
$$

Where, $U$ is mean velocity in vertical. Formula (3) could be written as:

$$
u=e^{A_{3}} \frac{\kappa U}{\ln \left(4 h / z_{0}\right)-8 / 3}\left(\frac{z}{h}\right)^{-A_{1}} z^{A_{2}}
$$

Parameters in formula (5) are determined by using nearshore measured data and the formula works best and the coefficient of correlation reaches 0.985 when $z_{0}, A_{1}, A_{2}$ and $A_{3}$ equal $0.0025,-0.02457,0.159018$ and 2.65953 respectively. Then formula (5) could be written as:

$$
u=K\left(\frac{z}{\kappa h}\right)^{\frac{9}{49}} U
$$

Where, $K=\frac{4.83}{\ln (1600 h)-8 / 3} h^{\frac{3}{19}}$

\section{VALIDATION AND COMPARISON OF VELOCITY PROFILE FORMULAE}

Apply formula (6) in Jiangsu Sea and compare the results with Soulsby formula (formula 7) which is widely used:

$$
u= \begin{cases}\left(\frac{z}{0.32 h}\right)^{\frac{2}{7}} U & 0<\frac{z}{h}<0.5 \\ 1.07 U & 0.5<\frac{z}{h}<1\end{cases}
$$

Measured data from 22 stations are used to verify formula (6) and Souslby formula. Figure 1 shows the comparison between calculation values of formula (6) and measured data, and figure 2 shows the comparison between Souslby formula and measured data. The figures show that the new formula is better than Soulsby formula in coefficient of correlation, and its distribution is more centralized.

For further comparison and analysis, data of 6 moments of 7 stations are chosen. In the figure, "-" is tide level hydrograph, and " 0 " is tide level.

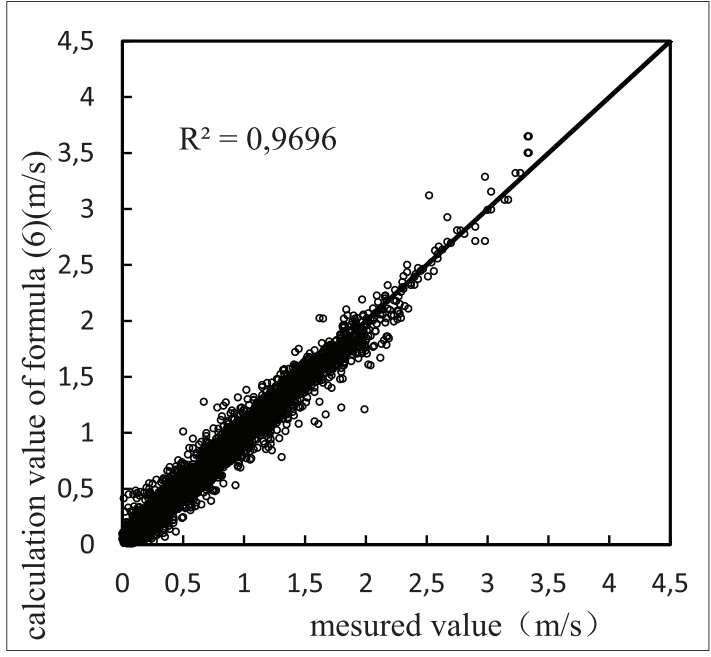

Fig.1 Comparison of formula (6) with measured data

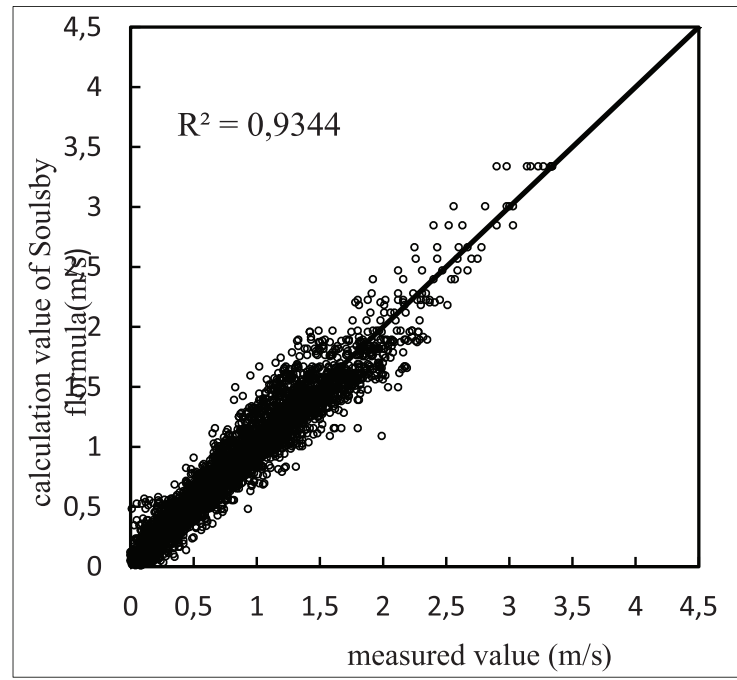

Fig.2 Comparison of Soulsby formula with measured data

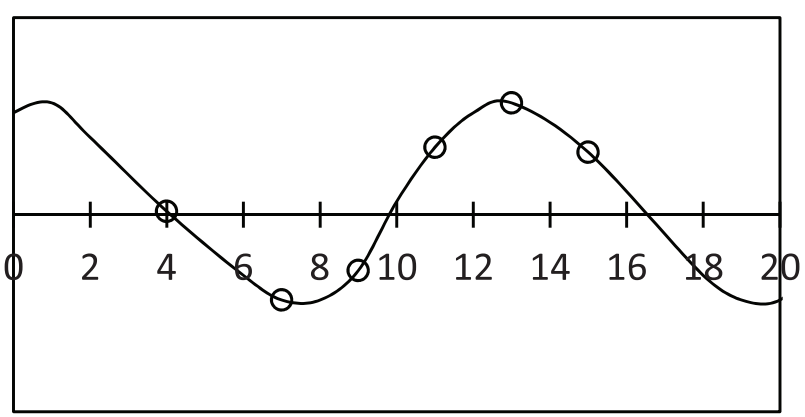

Figure 3 chosen moments for velocity profile verification

Figure 4-10 show comparison between formula (6), Soulsby formula and measured data. The chosen moments are arranged in time order from left to right. " 0 ” stands for measured data of velocity, "-_" for calculation value of formula (6) and “- . _" for that of Soulsby formula. Horizontal ordinate stands for $\frac{u}{U}$ and vertical coordinate for $\frac{z}{h}$. 
These figures show in different flow regime formula (6) can always reflect the characteristics of velocity profile of different stations. Compared with Soulsby formula, formula (6), a continuous function, works better, and it revises the situation where velocity is fixed when water depth is larger than $0.5 h$.
The comparison shows that formula (6) is a continuous function which is similar to Soulsby formula in form but simpler and easier to engineering use. In formula (6) friction velocity in form of Prandtl is introduced to reflect the impact of turbulence caused by bottom shear stress to the whole flow field. Formula (6) which does not need the maximum velocity and only needs the mean velocity in vertical, with more definite
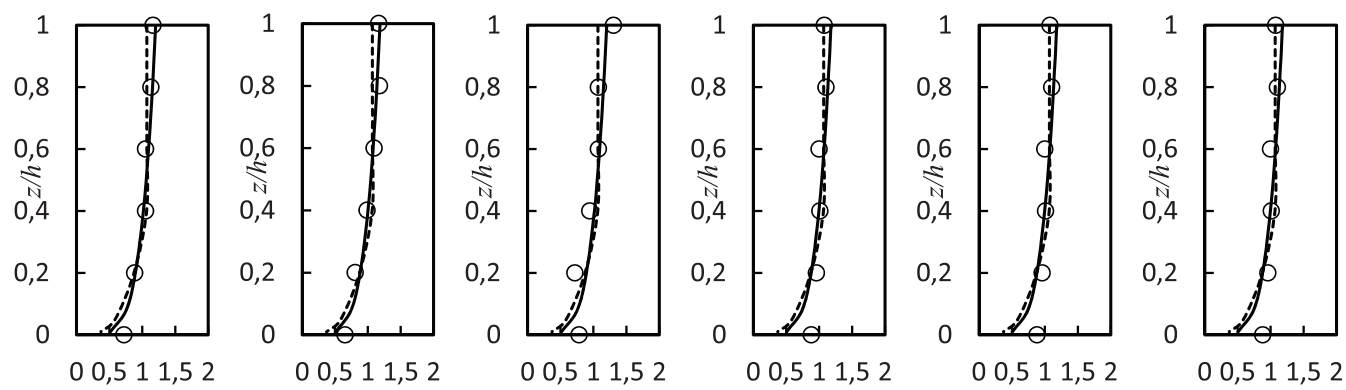

Fig.4 Comparison between formula (6), Soulsby formula and measured data of No.1 station
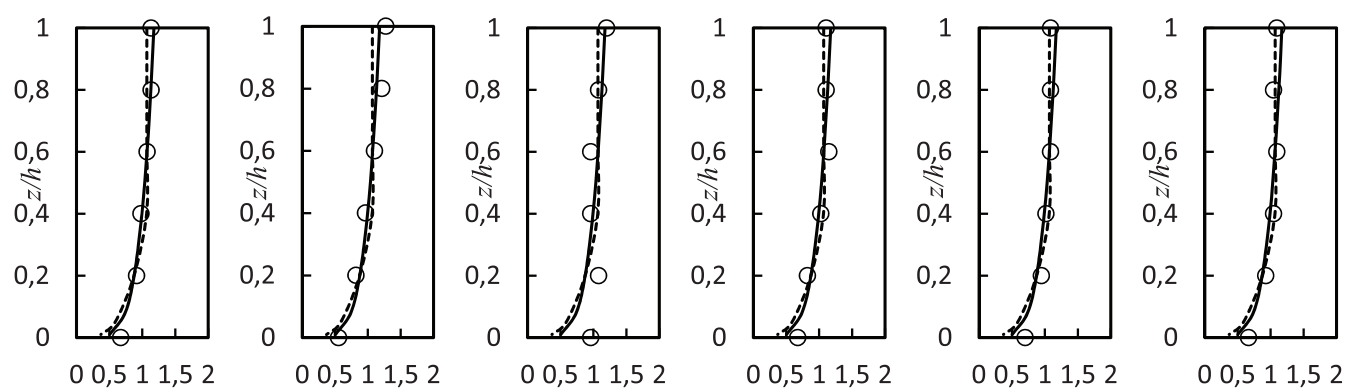

Fig.5 Comparison between formula (6), Soulsby formula and measured data of No.2 station
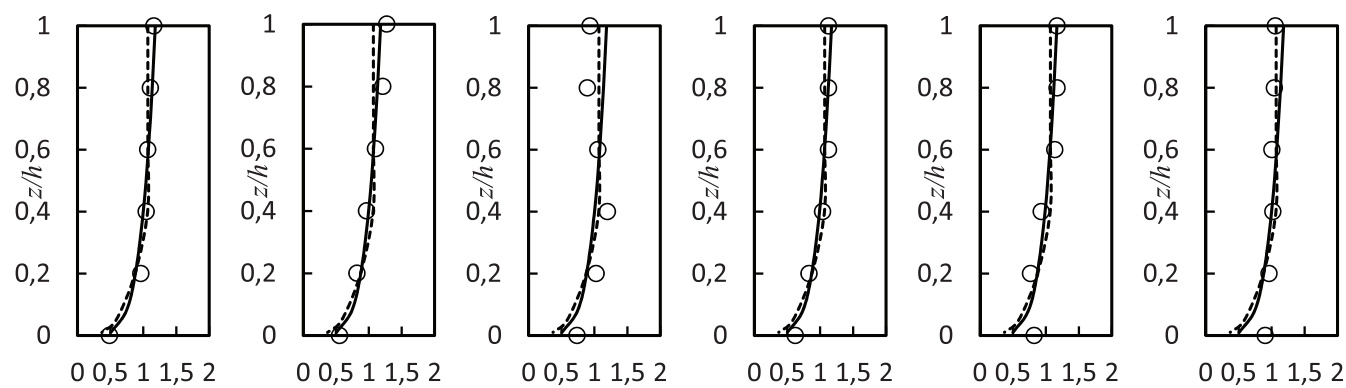

Fig.6 Comparison between formula (6), Soulsby formula and measured data of No.3 station
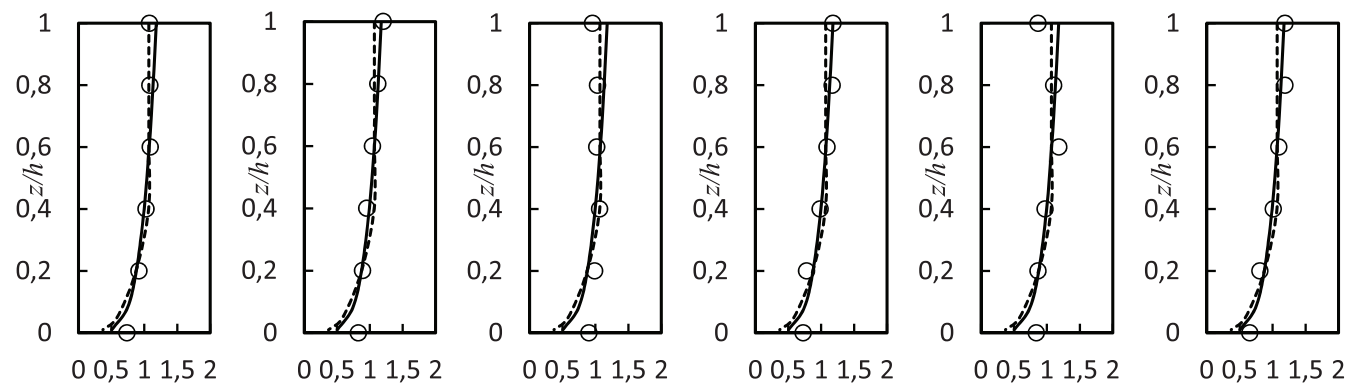

Fig.7 Comparison between formula (6), Soulsby formula and measured data of No.4 station 

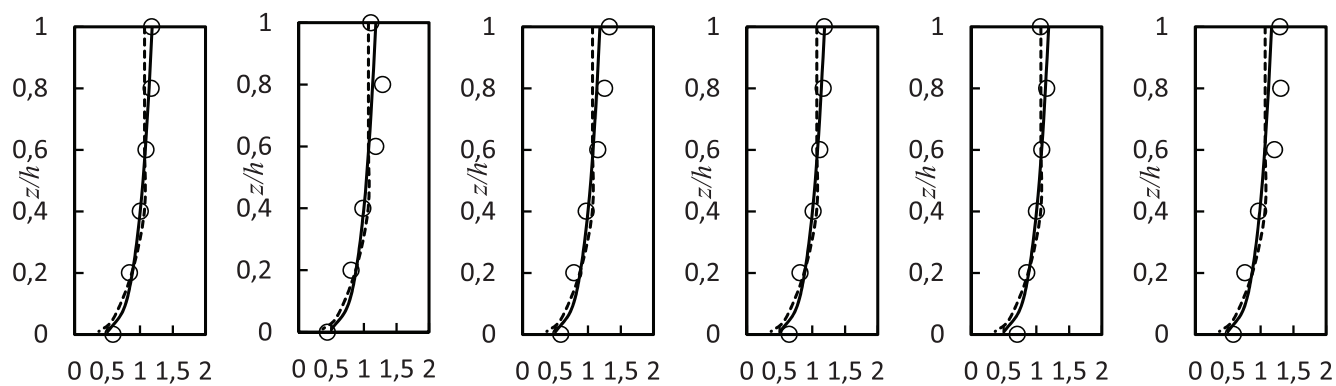

Fig.8 Comparison between formula (6), Soulsby formula and measured data of No.5 station
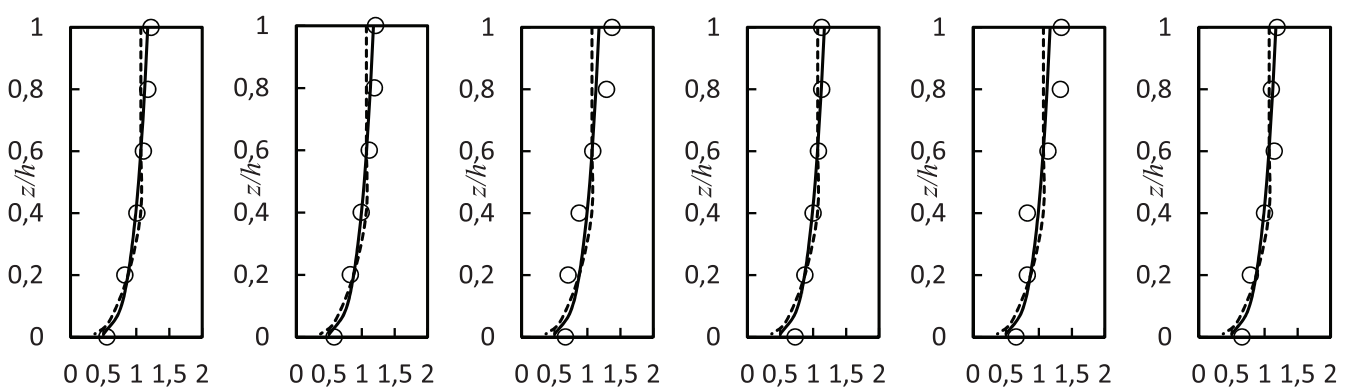

Fig. 9 Comparison between formula (6), Soulsby formula and measured data of No.6 station
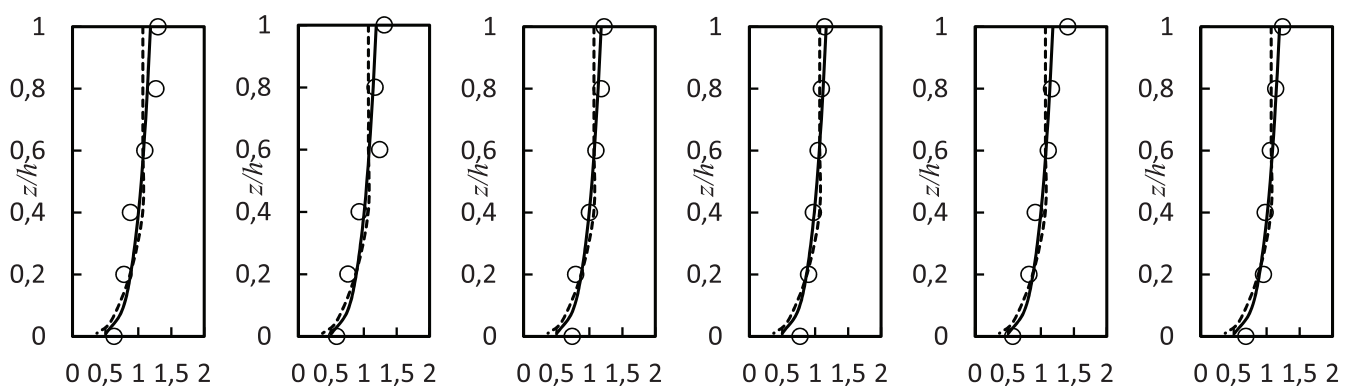

Fig.10 Comparison between formula (6), Soulsby formula and measured data of No.7 station

physical meanings, could show the influences of different flow properties on velocity profile by adjusting Karman constant. In field measurement, it is very difficult to get the maximum velocity within reasonable error control, but getting the mean velocity in vertical is much easier, which gives formula (6) higher applicability. The comparison between formula (6) and Soulsby formula is given in figure 2 .

\section{CONCLUSION}

In this paper, a new formula of velocity profile which is similar to that of Soulsby but simpler in form and easier to use is constructed. The new formula is a continuous function with specific physical meanings and verified with measured data, which shows the new formula has a high accuracy and can reflect the vertical distribution of flow velocity objectively. As to the estuary region with velocity profile in $\mathrm{S}$ form, this formula has some certain errors, which needs further study as well as the applicability of new formula in other sea areas.

Its advantages are as follows:

a. The new formula is a continuous function, convenient for engineering application and could be used for further study of vertical distribution of suspended sediment;

b. Friction velocity in form of Prandtl is introduced to the new formula to reflect the influences of bottom shear stress to the whole flow field, and Karman constant is used to reflect the influence of different flow regime to velocity profile with specific physical meaning.

c. Compared with logarithmic form, the new formula does only needs the mean velocity in vertical instead of both maximum velocity and mean velocity in vertical, which gives it better engineering practicability.

Apple the new formula in Jiangsu Sea and compare the results with that of Soulsby whose results show the new one has higher accuracy and can reflect the vertical distribution of flow velocity objectively.

As to the estuary region with velocity profile in $S$ form, this formula has some certain errors, which needs further study. 


\section{ACKNOWLEDGMENTS}

Funded By: the national natural science foundation of China (No.41276017).

\section{REFERENCES}

1. Fang Guo-hong, Takashi Ichiye. On the vertical structure of tidal currents in a homogeneous sea [J]. Geophysical Journal of the Royal Astronomical Society, 1983, 73 (1): 65-82.

2. He Bing-ju, Hao Jia-ling. Fractal Study of Vertical Flow Velocity Distribution of Yangtze River Estuary [J]. Water Resources and Power, 2015, 33(12):37-39+31.

3. Kumbhakar M, Ghoshal K. Two dimensional velocity distribution in open channels using Renyi entropy[J]. Physica A: Statistical Mechanics and its Applications, 2016.

4. Li Rui-jie, Feng Qing, Zheng Jun, Jiang Sen-hui. Analysis on vertical distribution of nearshore fluid velocity and sediment concentration [J]. Marine Science Bulletin, 2012, 31(6):607-612.

5. MONIN A S, YAGLOM A M. Stastical fluid mechanics : mechanics of turbulence [M]. Third Edition. Cambridge, MA: The MIT Press, 1977.

6. Ni Jin-ren, Wang Guang-qian, Zhang Hong-wu. The basic theory of solid-liquid two phrase flow and its new application [M]. Beijing: Science Press, 1991.

7. Ning Chien, Wang Zhao-hui. Mechanics of sediment transport [M]. Virginia:ASCE Press, 1999.

8. SCHAUER U. Determination of bottom boundary layer parameters at two shallow sea sites using the profile method [J]. Continental shelf Research, 1987, 7(10): 1211-1230.

9. Song Zhi-yao, Yan Yi-xin, Hao Jia-ling, et al. Study on the log-linear velocity profile of near-bed tidal current in estuarine and coastal waters [J]. China Ocean Engineering, 2006, 20(4): 573-584.

10. Mattheus, CR. Climate-Induced Changes in Rates of Headland-Beach Progradation along the Southern Coast of Lake Erie [J]. Journal of Coastal Research, 2014, 30(4): 743-755.

11. Soulsby R L. Dynamics of marine sands $[\mathrm{M}]$. London: Thomas Telford, 1997.

12. Soulsby R L. Tidal -current boundary layer in the sea Ocean Engineering Science [M], Wiley, New York, 1990.
13. TAYLOR P A, DYER K R. Theoretical models of flow near the bed and their implications for sediment transport [R]. New York: Wiley-Inter science, 1977.

14. Van Rijn. Principles of sediment transport in river, estuaries and coastal seas [M]. Amsterdam: Aqua Publication, 1993.

15. Jakubowski, M. Influence of pitting corrosion on fatigue and corrosion fatigue of ship and offshore structures, part II: load - PIT -crack interaction [J]. Polish Maritime Research. 2015, 22(3): 57-66.

16. Xiao Qian-lu, Li Rui-jie. Study of vertical velocity distribution calculation of tidal current in Xiyang Channel of radial sandbanks in South Yellow Sea of China [J]. Yangtze River, 2015, 46(3): 9-13.

\section{CONTACT WITH THE AUTHOR}

Li Chunhui

lichunhui35@126.com

Key Laboratory of Coastal Disaster and Defense Ministry of Education Hohai University, Nanjing 210098,

Environment Marine Laboratory Hohai University, Nanjing

CHINA 